\title{
The P450 side-chain cleavage enzyme Cyp11a2 facilitates steroidogenesis in zebrafish
}

\author{
Nan Li ${ }^{1,2}$, James A Oakes ${ }^{1,2}$, Karl-Heinz Storbeck ${ }^{3}$, Vincent T Cunliffe ${ }^{2,4, *}$, and Nils P Krone ${ }^{1,2,5,{ }^{*}}$ \\ 1Department of Oncology \& Metabolism, School of Medicine, University of Sheffield, Sheffield, UK \\ ${ }^{2}$ The Bateson Centre, Firth Court, Western Bank, Sheffield, UK \\ ${ }^{3}$ Department of Biochemistry, Stellenbosch University, Stellenbosch, Matieland, South Africa \\ 4Department of Biomedical Science, Firth Court, Western Bank, Sheffield, UK \\ ${ }^{5}$ Department of Medicine III, University Hospital Carl Gustav Carus, Technische Universität Dresden, Dresden, Germany \\ Correspondence should be addressed to N P Krone: n.krone@sheffield.ac.uk \\ *(V T Cunliffe and N P Krone contributed equally to this work)
}

\begin{abstract}
Cytochrome P450 side-chain cleavage enzyme, encoded by the CYP11A1 gene, catalyzes the first and rate-limiting step of steroid hormone biosynthesis. Previous morpholinoknockdown studies in zebrafish suggested cyp11a2 is a functional equivalent of human CYP11A1 and is essential for interrenal steroidogenesis in zebrafish larvae. The role of Cyp11a2 in adult zebrafish, particularly in gonadal steroidogenesis, remains elusive. To explore the role of Cyp11a2 in adults, we developed zebrafish mutant lines by creating deletions in cyp 11a2 using the CRISPR/Cas9 genomic engineering approach. Homozygous cyp11a2 mutant zebrafish larvae showed an upregulation of the hypothalamic-pituitaryinterrenal axis. Furthermore, these Cyp11a2-deficient zebrafish demonstrated profound glucocorticoid and androgen deficiencies. Cyp11a2 homozygotes only developed into males with feminized secondary sex characteristics. Adult cyp11a2-/- mutant fish showed a lack of natural breeding behaviors. Histological characterization revealed disorganized testicular structure and significantly decreased numbers of mature spermatozoa. These findings are further supported by the downregulation of the expression of several pro-male genes in the testes of cyp11a2 homozygous zebrafish, including sox $9 a$, dmrt 1 and amh. Moreover, the spermatogonia markers nanos 2 and piwil1 were upregulated, while the spermatocytes marker sycp3 and spermatids marker odf3b were downregulated in the testes of cyp11a2 homozygous mutants. Our expression analysis is consistent with our histological studies, suggesting that spermatogonia are the predominant cell types in the testes of cyp11a2 homozygous mutants. Our work thus demonstrates the crucial role of Cyp11a2 in interrenal and gonadal steroidogenesis in zebrafish larvae and adults.
\end{abstract}
Key Words
- Cyp11a2
- glucocorticoid
- androgen
- zebrafish
- CRISPR/Cas9

\section{Introduction}

Zebrafish has been established as vertebrate model to elucidate gene function in development and disease. Despite their evolutionary distances, zebrafish share extensive conservation with humans at both genomic and functional levels. In particular, molecular pathways controlling steroidogenesis and steroid signaling are highly conserved (Lohr \& Hammerschmidt 2011, Tokarz et al. 2013, 2015). Similar to humans, cortisol is 
the main glucocorticoid in zebrafish and is produced in the interrenal (equivalent of the mammalian adrenal gland) (Dickmeis 2009). The production of cortisol is under the control of hypothalamus and pituitary, forming the hypothalamus-pituitary-interrenal (HPI) axis (Liu 2007, To et al. 2007). In addition to the interrenal, steroidogenesis in zebrafish also occurs in other tissues such as gonads and brain (Tokarz et al. 2013).

The cytochrome P450 side-chain cleavage enzyme, encoded by the CYP11A1 gene, catalyzes the first and ratelimiting step of steroidogenesis and is a prerequisite for all steroid hormone biosynthesis (Miller \& Auchus 2011). Previous studies identified two cyp11a genes in zebrafish, cyp11a1 and cyp11a2 (Goldstone et al. 2010, Parajes et al. 2013). Our previous work assumed a paralog-specific role for each cyp11a gene in zebrafish larvae (Parajes et al. 2013). During early development, cyp11a1 is expressed from zygote to segmentation periods (22-24 hpf, hours post fertilization), while the expression of cyp11a2 becomes prominent just before the onset of de novo (48 hpf) and HPI-mediated (96 hpf) cortisol biosynthesis. Morpholino-mediated mRNA knockdown revealed that cyp11a1 is required for early embryonic development, whereas its paralog cyp11a2 represents the functional equivalent of human CYP11A1 and is essential for interrenal steroidogenesis in larvae (Parajes et al. 2013).

However, loss-of-function studies using morpholinos only transiently reduce expression levels of target genes, and the physiological role of Cyp11a2 in adult zebrafish remains unclear. To define the function of Cyp11a2 in adult zebrafish, we developed zebrafish cyp11a2-null alleles using a CRISPR/Cas9 approach. Cyp11a2-deficient zebrafish had a phenotype resembling severe combined interrenal and gonadal insufficiency, including decreased expression of glucocorticoid target genes, altered steroid hormone profiles, reduced fertility, disorganized gonadal tissues and all-male fish with feminized secondary sex characteristics. Thus, we demonstrate that cyp11a2 is the functional P450 side-chain cleavage enzyme paralog in zebrafish, facilitating the primary step of interrenal and gonadal steroidogenesis.

\section{Materials and methods}

\section{Zebrafish maintenance}

The zebrafish strains were maintained in a recirculating system (ZebTECTM, Techniplast ${ }^{\circledR}$, Kettering, UK and Sheffield, UK) at $28.5^{\circ} \mathrm{C}$ in a 10 -h dark, 14-h light photoperiod. Embryos were obtained by natural spawning and incubated at $28.5^{\circ} \mathrm{C}$ in $\mathrm{E} 3$ medium $(5 \mathrm{mmol} / \mathrm{L} \mathrm{NaCl}$, $0.17 \mathrm{mmol} / \mathrm{L} \mathrm{KCl}, 0.33 \mathrm{mmol} / \mathrm{L} \mathrm{CaCl}_{2}, 0.33 \mathrm{mmol} / \mathrm{L} \mathrm{MgSO}_{4}$ ) containing $0.1 \%(\mathrm{v} / \mathrm{v})$ methylene blue. The developmental stages were determined according to hours post fertilization (hpf), and days post fertilization (dpf). All procedures were approved by the United Kingdom Home Office and carried out in line with the Animals (Scientific Procedures) Act 1986.

\section{Generation of cyp11a2 mutants by CRISPR/Cas9}

Gene-specific guide RNA (gRNA) was designed against an optimal CRISPR site targeting exon 5 of cyp11a2 (ENSDARG00000092696) following an adapted strategy described previously (Hruscha et al. 2013, Talbot \& Amacher 2014). gRNA antisense DNA sequence was first amplified using $2.5 \mu \mathrm{L}$ gRNA primer $1(10 \mu \mathrm{M}$, 5'-GCGTAATACGACTCACTATAG-3'), $2.5 \mu \mathrm{L}$ gRNA primer 2 (10 $\mu \mathrm{M}, \quad 5^{\prime}$-AAAAAAGCACCGACTCGGTGCCAC-3'), $2 \mu \mathrm{L}$ gRNA guide oligo $(1 \mu \mathrm{M}$, oligonucleotide sequence is listed in Supplementary Table 1 , see section on supplementary materials given at the end of this article), $20 \mu \mathrm{L} 5 \times$ FIREPol $^{\circledR}$ Master Mix (Solis Biodyne, Tartu, Estonia), and $73 \mu \mathrm{L}$ MilliQ $\mathrm{H}_{2} \mathrm{O}$. The whole PCR product was loaded on a $2.5 \%(\mathrm{w} / \mathrm{v})$ agarose gel and extracted using MinElute Gel Extraction Kit (Qiagen) following the manufacturer's protocol. The subsequent in vitro transcription was performed using MEGAshortscript T7 kit (Life Technologies), using $1 \mu \mathrm{L}$ as a template. Fertilized eggs were injected at one-cell stage with $1 \mathrm{~nL}$ of a solution containing $2 \mu \mathrm{M}$ Cas9 protein (NEB, Hitchin, UK), $2.4 \mathrm{ng} / \mathrm{nL}$ gRNA and $0.1 \%$ (v/v) phenol red. Genomic DNA was extracted from injected individuals at $5 \mathrm{dpf}$ to verify the presence of mutations and confirm the activity of the gRNA. Injected embryos were raised to adulthood (F0 generation). To confirm germline transmission of the mutation, a pool of 20 embryos collected from out-crosses between the F0 and wild-type were genotyped. F0 fish with successful germline transmission were kept as F0 founders. Embryos from out-crosses were raised to generate the F1 generation and genotyped for heterozygous mutations using fin clips. F1 heterozygotes were then out-crossed to generate the F2 heterozygotes which were in-crossed to characterize the cyp11a2 mutations.

\section{Genotyping cyp11a2 mutants}

Adult fish were anesthetized in tricaine methanesulfonate (MS222, Sigma-Aldrich) and one-third of caudal fin was 
cut with a sharp blade. Genomic DNA was extracted from clipped caudal fin tissue or from whole larvae. Samples were lysed in $20 \mu \mathrm{L}$ of $50 \mathrm{mM} \mathrm{NaOH}$ and boiled for $10 \mathrm{~min}$ at $98^{\circ} \mathrm{C}$ and $1 / 10$ volume of $100 \mathrm{mM}$ Tris- $\mathrm{HCl}$ pH8.0 was added to buffer the reaction. PCR amplification of cyp11a2 exon 5 was carried out in $20 \mu \mathrm{L}$ reaction with $1 \mu \mathrm{L}$ genomic DNA template, $1 \mu \mathrm{L}$ forward primer $(10 \mu \mathrm{M}$, 5'-TTTAAGACCACCTCGCCCAT- ${ }^{\prime}$ ) and reverse primer (10 $\mu \mathrm{M}, \quad$ 5'-GAGCCAGATTCAAACCAGCA-3') using FIREPol $^{\circledR}$ Master Mix system (Solis Biodyne). The PCR product was analyzed on a $2.5 \%(\mathrm{w} / \mathrm{v})$ agarose gel for any indel mutations.

\section{Analysis of visual background adaptation (VBA)}

To identify cyp11a2-/- mutants for defective VBA behavior, larvae were analyzed at $96 \mathrm{hpf}$ as described previously (Griffin et al. 2016). Briefly, larvae were maintained in a dark environment for at least $60 \mathrm{~min}$, followed by exposure to bright illumination for $30 \mathrm{~min}$. Larvae were then scored using a stringent criterion for lack of melanophore contraction, as an indicator of defective VBA. Only larvae with strongly dark pigmentation were designated as VBA-negative (VBA-) while only the lightest ones were designated as VBA-positive (VBA+) (Fig. 1C). Larvae with

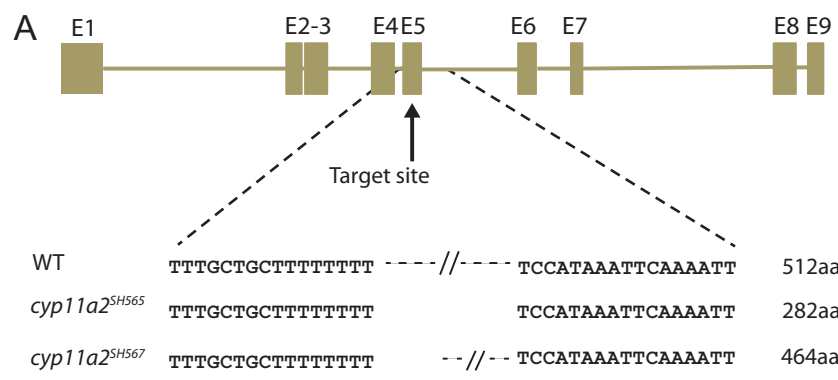

B

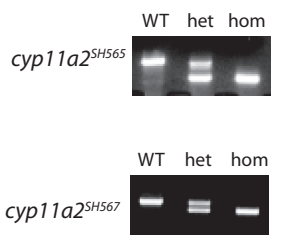

C

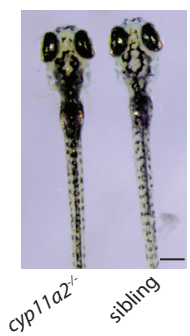

512 aa 282aa $464 a a$

Generation of cyp11a2 null-allele using CRIPSR/Cas9 strategy. (A) Exon 5 of cyp11a2 was targeted for genetic disruption by CRISPR/Cas9.

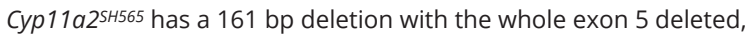
resulting in a truncated protein of 282aa. Cyp11a2 ${ }^{\text {SH567 }}$ has a deletion of $144 \mathrm{bp}$ leading to a truncated protein of 464aa. (B) Gel electrophoresis shows genotyping of wild-type, heterozygotes and homozygotes by PCR. (C) cyp 11a2-/- mutant larvae (120 dpf) had impaired visual-mediated background adaption. Scale bar: $300 \mu \mathrm{m}$. ambiguous pigmentation level were discarded. The groups of larvae with either dark or light pigmentation were then genotyped to confirm the accuracy of VBA analysis.

\section{Steroid hormones measurements by LC-MS/MS}

Since over 90\% VBA- larvae were confirmed to be cyp11a2-/- mutants (Supplementary Table 2), we used this method to identify homozygous mutants and wild-type siblings for steroid hormone measurements in larvae. Three clutches of $150 \mathrm{VBA}+$ larvae and $150 \mathrm{VBA}-$ larvae were collected at $120 \mathrm{hpf}$ into a silanized tube and snap frozen on dry ice. $1 \mathrm{~mL}$ of $1 \times$ PBS was added to the samples, and the samples were lysed through four rounds of freezethawing. After lysis the samples were homogenized with a pestle homogenizer and freeze-dried. The whole bodies of adult wild-type siblings and cyp11a2-/- mutants at $180 \mathrm{dpf}$ (eight biological replicates each) were snap frozen on dry ice. The adult samples were homogenized using a Mikro-Dismembrator S (Sartorius, Gottingen, Germany) and then freeze-dried. Approximately $100 \mathrm{mg}$ of the dried samples were transferred to a glass test tube and resuspended in $900 \mu \mathrm{L}$ MilliQ water and $100 \mu \mathrm{L}$ MilliQ water containing internal standard (15 ng D4-cortisol, D7-11ß-hydroxyandrostenedione, D7-androstenedione and $1.5 \mathrm{ng}$ D2-testosterone). The steroids were extracted twice using $3 \mathrm{~mL}$ methyl tert-butyl ether (MTBE). Following centrifugation for $5 \mathrm{~min}$ at low speed the MTBE fractions for each sample were pooled and dried under a stream of nitrogen at $45^{\circ} \mathrm{C}$. The dried residue was resuspended in $150 \mu \mathrm{L} 50 \%(\mathrm{v} / \mathrm{v})$ methanol prior to analysis. Steroids were separated and quantified using an Acquity UPLC System (Waters, Milford, CT, USA) coupled to a Xevo TQ-S tandem mass spectrometer (Waters) as previously described (O'Reilly et al. 2017).

\section{Induction of osmotic stress}

Three clutches of $25 \mathrm{VBA}+$ and VBA- larvae were collected at $120 \mathrm{hpf}$. Larvae were treated in $250 \mathrm{mM}$ sodium chloride (in E3 medium) for 20 min. Subsequently, larvae were washed and incubated in E3 medium for $4 \mathrm{~h}$ before sampling for RNA extraction (Griffin et al. 2016).

\section{Comparative gene expression analysis by quantitative real-time PCR (qRT-PCR)}

Three clutches of $25 \mathrm{VBA}+$ and VBA- larvae were collected at 120 hpf. Gonad, pituitary and liver were collected from 3 to 5 male adult individuals. All the samples were 
snap frozen on dry ice before processing. Total RNA from whole larvae or adult tissues and RT were performed as previously described (Griffin et al. 2016). Oligonucleotide sequences are listed in Supplementary Table 3. The qRT-PCR reaction was performed in $10 \mu \mathrm{L}$ volumes on 384-well plates using PowerUP SYBR Green Master Mix (Applied Biosystems) on a 7900HT Fast Real-Time PCR System (Applied Biosystems). Expression levels for each gene were normalized to reference gene $\beta$-actin, and fold changes were calculated relative to wild-type siblings. Expression analysis of pomca, $f \operatorname{sh} b, l h b, f s h r, l h c g r, g h 1$ was performed using TaqMan Universal PCR Master Mix and TaqMan Gene Expression Assays (Applied Biosystems). Expression level was then normalized to reference gene ef1a.

\section{Whole-mount RNA in situ hybridization (WISH)}

Whole-mount RNA in situ hybridization (WISH) was carried out following a standard protocol (Thisse \& Thisse 2008). Briefly, 40 wild-type siblings and 40 cyp11a2-/- mutant larvae at $5 \mathrm{dpf}$ were fixed in $4 \%(\mathrm{w} / \mathrm{v})$ paraformaldehyde (PFA) in $1 \times$ PBS overnight and the next day were hybridized with pomca probe at $70^{\circ} \mathrm{C}$ overnight. Larvae were then incubated with anti-digoxigenin-AP antibody solution (1:4000) overnight at $4^{\circ} \mathrm{C}$ and stained with NBT/BCIP until it reached the desired intensity. The stained larvae were imaged using Leica DFC420 camera (Leica Camera AG). The pomca probe was generated as previously described (Muthu et al. 2016).

\section{Morphological analysis of adults}

The gross morphology of wild-type siblings and cyp11a2-/- mutants were compared at $180 \mathrm{dpf}(n=10$ for each genotype). The secondary sex characteristics were examined based on a well-established method involving body shape and coloration (Schilling 2002, Dranow et al. 2013). The characterization was further confirmed by other two independent researchers blinded to the genotype. The weight and length of adult zebrafish were measured after being killed. Subsequently, zebrafish were dissected, gonads were examined and photographed.

\section{Breeding ability assessment and in vitro fertilization (IVF)}

A cyp11a2-/- mutant male or a wild-type sibling male was out-crossed to an unrelated wild-type female in a breeding tank with a divider in the middle on the afternoon prior to a breeding trial. The following morning, the divider separating male and female fish was removed and they were allowed to mate. The number of fertilized eggs was counted after $3 \mathrm{~h}$.

For IVF experiments, sperm were collected by dissecting the testes of killed males, which were then homogenized in $50 \mu \mathrm{L}$ Hank's balanced salt solution prepared as previously described (Kroeger et al. 2014). Eggs were collected from anesthetized wild-type females by gentle palpation of the abdomen. $20 \mu \mathrm{L}$ of sperm solution was mixed with the clutch of collected eggs, followed by $1 \mathrm{~mL}$ of $\mathrm{E} 3$ medium to activate the sperm. After 2 min, further E3 medium was added and eggs were incubated at $28.5^{\circ} \mathrm{C}$ for development (Westerfield 2000). Fertilization of eggs was confirmed by visualization under a dissecting microscope.

\section{Mating behavior analysis}

A cyp11a2-- mutant male or wild-type male sibling was paired with a wild-type female in the late afternoon with a divider in the middle of the breeding tank. The next morning, the divider was removed and breeding behaviors was recorded from above the tank using the Zebralab software (Viewpoint Life Sciences, Montreal, Canada). Videos of the breeding behaviors were then analyzed. The frequency and duration of intimate contacts, including parallel swimming, chasing and spawning, were recorded.

\section{Sperm counting}

Sperm were collected by dissecting the testes of killed males, followed by homogenization in a $100 \times$ mass:volume dilution with 600 mosmol/kg Hank's balanced salt solution prepared as described (Jing et al. 2009). $10 \mu \mathrm{L}$ of sperm solution was transferred to each counting chamber of a dual-chamber Improved Neubauer hemocytometer (Hawksley, Lancing, UK) and sperm from each sample were counted in duplicate according to the protocol specified in WHO Laboratory manual for the examination and processing of human semen (World Health Organization 2010). A minimum of 200 sperms were counted for each replicate. Sperm concentration was determined by calculating the concentration per nanolitre and multiplying this by the weight to volume dilution factor. The gonadosomatic index (GSI) was calculated using the formula (gonad weight/total tissue weight) $\times 100$. https://joe.bioscientifica.com

https://doi.org/10.1530/JOE-19-0384
(C) 2020 Society for Endocrinology Published by Bioscientifica Ltd. Printed in Great Britain 


\section{Hematoxylin and eosin (H\&E) staining}

Adult fish were fixed in $4 \%(\mathrm{w} / \mathrm{v})$ paraformaldehyde (PFA) in $1 \mathrm{x}$ PBS for 4 days at $4^{\circ} \mathrm{C}$ followed by brief washing in $1 \times$ PBS. Fish were decalcified in $0.25 \mathrm{M}$ EDTA pH 8.0 for 4 days at room temperature and then transferred to $70 \%$ $(\mathrm{v} / \mathrm{v})$ ethanol for storage at $4^{\circ} \mathrm{C}$. The head, caudal fins and anal fins of the zebrafish were removed and the samples were transferred to a tissue processor (Leica TP2010) for dehydration and paraffin infiltration. After the preprocessing, the samples were embedded in paraffin wax and $5 \mu \mathrm{m}$ sections were cut through the entire gonad. For $H \& E$ staining, samples were dewaxed using xylene and rehydrated by transferring through a series of ethanol baths. Samples were then stained with Gill's hematoxylin for $1 \mathrm{~min}$ before dehydration in ethanol. Samples were stained with $1 \%$ eosin in $95 \%(\mathrm{v} / \mathrm{v})$ ethanol for $30 \mathrm{~s}$ and subsequently washed in absolute ethanol for three times. Samples were transferred to xylene and mounted using DPX mountant. The mounted samples were left dry for overnight and imaged using Leica DFC420 camera (Leica Camera AG).

\section{Statistical analysis}

Statistical analysis and graphics were performed using Graphpad Prism 7.0c (GraphPad Software). For the comparison of means between two samples, unpaired $t$-tests were used. For the comparison of means of more than two samples, one- or two-way ANOVA (ANOVA) and Sidak's multiple comparisons test was used.

\section{Results}

\section{Generation of cyp11a2-null alleles in zebrafish with CRISPR/Cas9}

To fully understand the function of Cyp11a2 in zebrafish larvae and adults, we disrupted the cyp11a2 gene employing a CRISPR/Cas9 strategy. gRNA was designed to target a $18 \mathrm{bp}$ site (AATATCTACAGACAATTG) in exon 5 for generation of a 5-prime disruption within the cyp11a2 gene. Genomic disruption in the germline of injected embryos was detected by conventional PCR. Sanger sequencing of the F1 generation identified three different heritable alleles within the targeted region. Two lines were subsequently maintained (cyp11a2sH565,

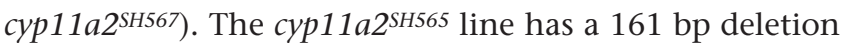

(c.818_978del) spanning the entire exon 5, introducing a frameshift with a premature stop at amino-acid position 282 (p.A273DfsX10) (Fig. 1A and B). The second-line cyp11a2 $2^{\text {SH567 }}$ carried a 144 bp deletion (c.821_964del), subsequently causing a 48 amino-acid in-frame deletion (p.274_321del) (Fig. 1A and B). Thus, both mutations are predicted to abolish Cyp11a2 activity.

The phenotypes of cyp11a2 homozygous mutant larvae were characterized before $5 \mathrm{dpf}$. cyp11a2sH565 and cyp11a2sH567 homozygous mutants were morphologically similar to control siblings during early development. The cyp11a2 ${ }^{\text {SH565 }}$ line was used for subsequent analysis and will be named cyp11a2-/- hereafter.

Background adaptation in zebrafish has been associated with impaired glucocorticoid biosynthesis and signaling (Griffin et al. 2016, Eachus et al. 2017). Larvae from a heterozygous in-cross were analyzed by VBA assessment at $96 \mathrm{hpf}$ and sorted into groups with either dark or light pigmentation (Fig. 1C). Subsequent genotyping revealed over $90 \%$ of VBA-negative larvae were cyp11a2-/- mutants (Supplementary Table 2). VBA-positive larvae were either wild-type or cyp11a2+-, confirming that cyp11a2-/- mutants are reliably identified by VBA assessment.

\section{Impaired steroid biosynthesis and systemic changes in cyp11a2-/- mutant larvae}

To assess the importance of Cyp11a2 in steroidogenesis, we measured cortisol concentrations by LC-MS/MS in cyp11a2-/- larvae. At $96 \mathrm{hpf}$, larvae from a heterozygous in-cross were sorted by VBA assessment into VBA+ and VBA- groups (150 larvae in each group, 3 replicates). Steroid hormone profiles were analyzed at $120 \mathrm{hpf}$. The cortisol concentration was significantly reduced in cyp11a2-/- mutants compared to their siblings $(P=0.0143)$ (Fig. 2A).

To further analyze the loss of Cyp11a2 on glucocorticoid action, qRT-PCR was used to assay the transcriptional responses of glucocorticoid-inducible genes after exposure to osmotic stress. Transcription of the glucocorticoid-responsive genes $f k b p 5(P<0.0001)$ and pck1 $(P=0.0135)$ was significantly decreased in cyp11a2-/mutant larvae compared to siblings, when maintained under basal conditions (Fig. 2B and C). Exposure to osmotic stress resulted in a significant increase in the expression of both $f k b p 5(P<0.0001)$ and $p c k 1 \quad(P=0.0088)$ in wild-types, but this treatment had no significant effect https://joe.bioscientifica.com https://doi.org/10.1530/JOE-19-0384 (c) 2020 Society for Endocrinology Published by Bioscientifica Ltd. Printed in Great Britain 
(n)
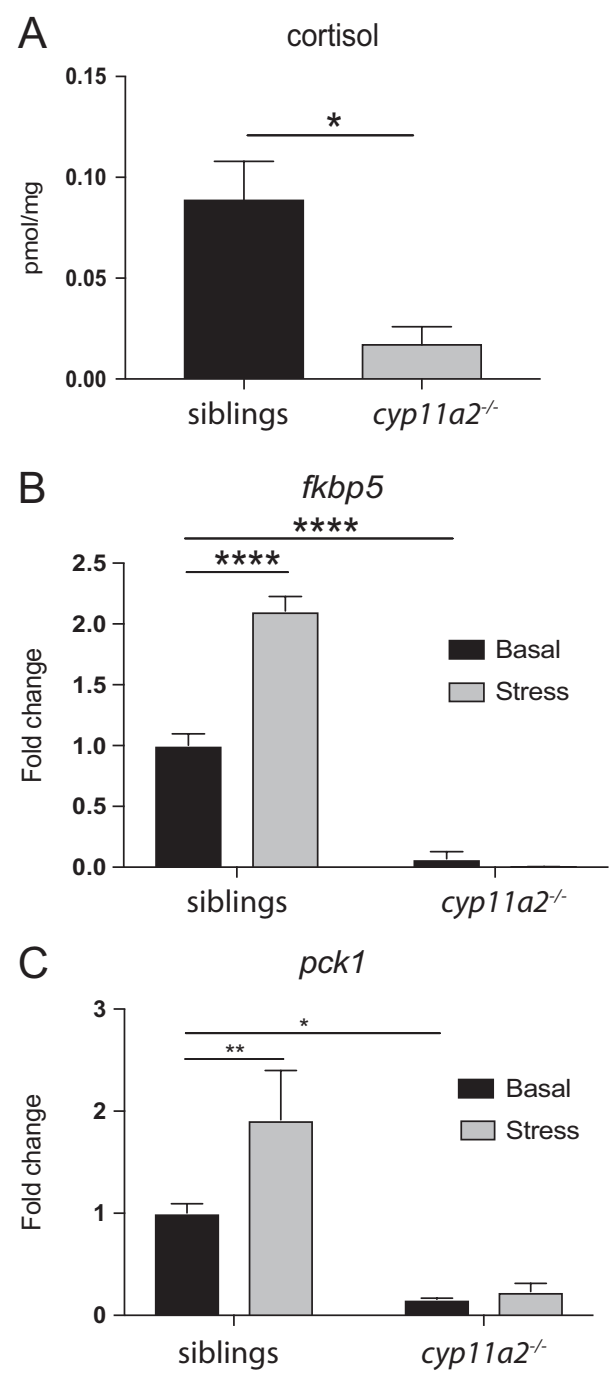

Figure 2

Zebrafish cyp11a2-/- mutant larvae are glucocorticoid deficient. (A) LC-MS/MS showed significantly reduced level of cortisol in cyp11a2 homozygous mutant larvae at $120 \mathrm{hpf}$ (unpaired $t$-test). Analysis of baseline and stress-induced transcript levels of (B) $f k b p 5$ and (C) $p c k 1$ using qRT-PCR in $120 \mathrm{hpf}$ siblings and homozygous larvae (two-way ANOVA). ${ }^{*} P<0.05 ;{ }^{* * P}<0.005 ; * * \star \star P<0.0001$.

on expression levels of these genes in cyp11a2-/- larvae (Fig. 2B and C).

Moreover, impaired cortisol biosynthesis led to significantly higher levels of pomca expression measured by qRT-PCR in cyp11a2-/- larvae $(P=0.001)$ (Fig. 3A), suggesting activation of the HPI axis due to lack of negative feedback. This finding was confirmed by WISH showing increased staining of a pomca probe in pituitary tissue in cyp11a2-/- larvae compared with wild-type siblings (Fig. 3B).

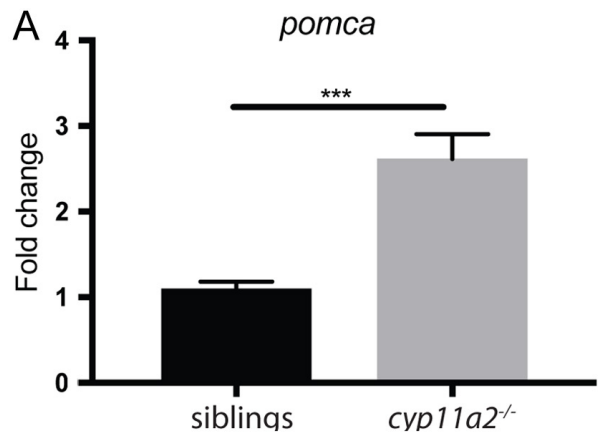

B

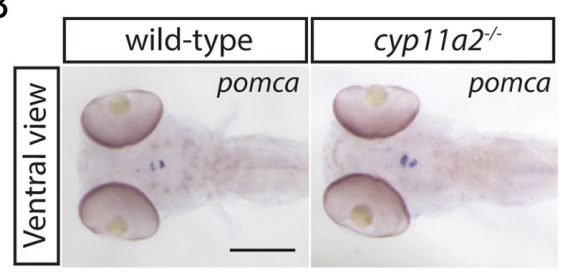

Figure 3

Zebrafish cyp11a2-/- mutants have a dysregulated HPI axis at $120 \mathrm{hpf}$. (A) qRT-PCR analysis of the expression of pomca was significantly upregulated in cyp 11a2-/- mutant larvae (unpaired $t$-test, $* \star \star P=0.001$ ). (B) WISH analysis showed the expression of pomca was increased in cyp11a2-/- mutant larvae compared to wild-type. Scale bar: $0.3 \mathrm{~mm}$.

\section{Cyp11a2 deficiency causes the development of only male fish with partly feminized secondary sex characteristics}

Despite their profound glucocorticoid deficiency, cyp11a2-/- zebrafish develop into viable adults. Typically, wild-type adult male zebrafish have a slender body shape with pinkish-yellow coloration, whereas wild-type females have an egg-filled abdomen of bluishsilver color (Schilling 2002, Dranow et al. 2013). The gross morphology of wild-type siblings and cyp11a2-/- mutants was compared at $180 \mathrm{dpf}$ ( $n=10$ for each genotype). All cyp11a2-/- animals had female-like bluish-silver body pigmentation and an abdomen rounder than that of wild-type males. Wild-type males had a dark yellow anal fin, compared to a light-yellow color of this structure in wild-type females. All cyp11a2-/- mutant animals had a dark yellow-pigmented anal fin. However, similar to wildtype females, cyp11a2-/- mutants displayed wider blue and narrower yellow stripes than wild-type males (Fig. 4A). Taken together, cyp11a2-/- mutants showed mixed secondary sex characteristics with more pronounced female secondary sex characteristics. Interestingly, the genital papilla was not apparent in all cyp11a2-/- fish, suggesting that their gonadal sex is male (Yossa et al. 2013). 
A

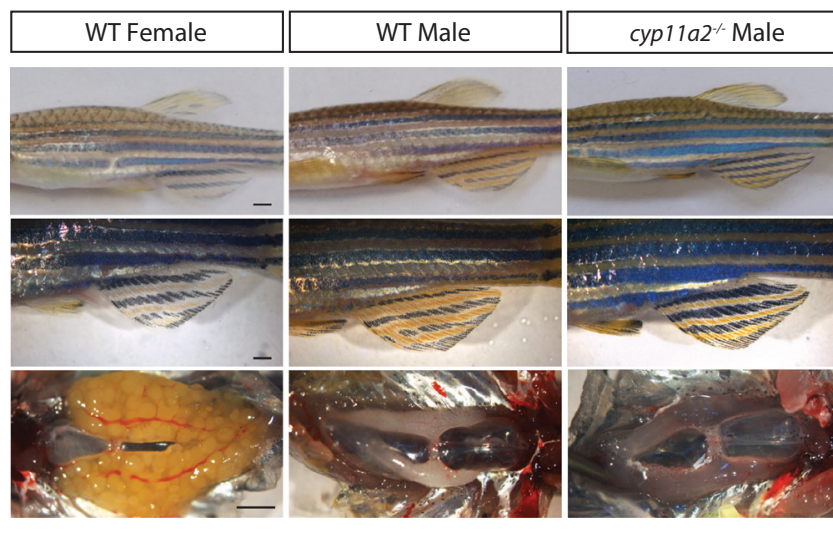

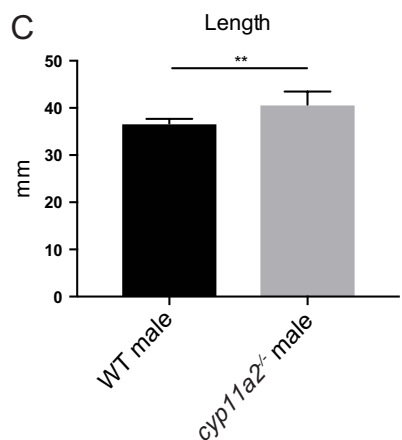
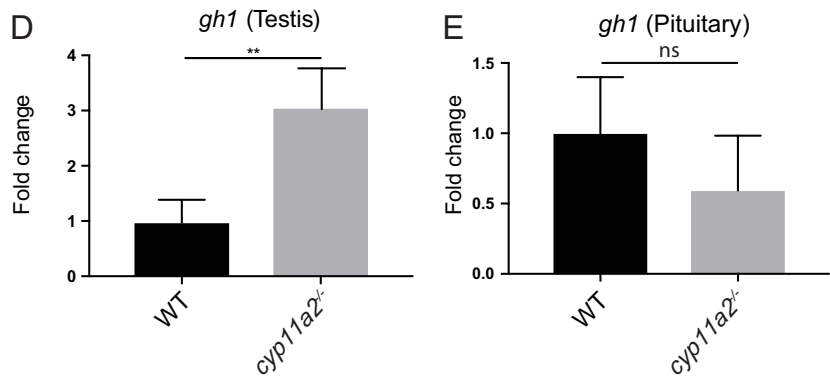

Figure 4

Depletion of Cyp11a2 leads to all-male homozygous fish. (A) Cyp11a2 deficiency caused fish to develop only into males as seen with the presence of testes and an apparent absence of the genital papilla. The

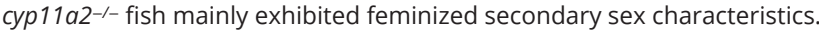
Scale bar: $1 \mathrm{~mm}$. Adult cyp11a2-/- zebrafish exhibited increased (B) weight and (C) length compared to wild-type siblings. (D) Expression of gh1 was significantly increased in the testes of cyp11a2-/- mutant adults compared to that of wild-type adult males $(n=5)$. (E) Expression of gh1 was unchanged in the pituitary of cyp11a2-/- mutant adults compared to wild-type adult males $(n=5)$. An unpaired $t$ test was used for the analysis. $\star P<0.05 ; * * P<0.005$

Further anatomical examination demonstrated that wild-type animals with male or female secondary sex characteristics possessed testes or ovaries at similar proportions $(n=20)$. By contrast, all cyp11a2-/mutants had testes and none had ovaries $(n=20)$ (Fig. $4 \mathrm{~A})$. Interestingly, cyp11a2-/- mutant fish were found to be significantly heavier $(P=0.0132)$ and longer $(P=0.001)$ than their wild-type male and female siblings
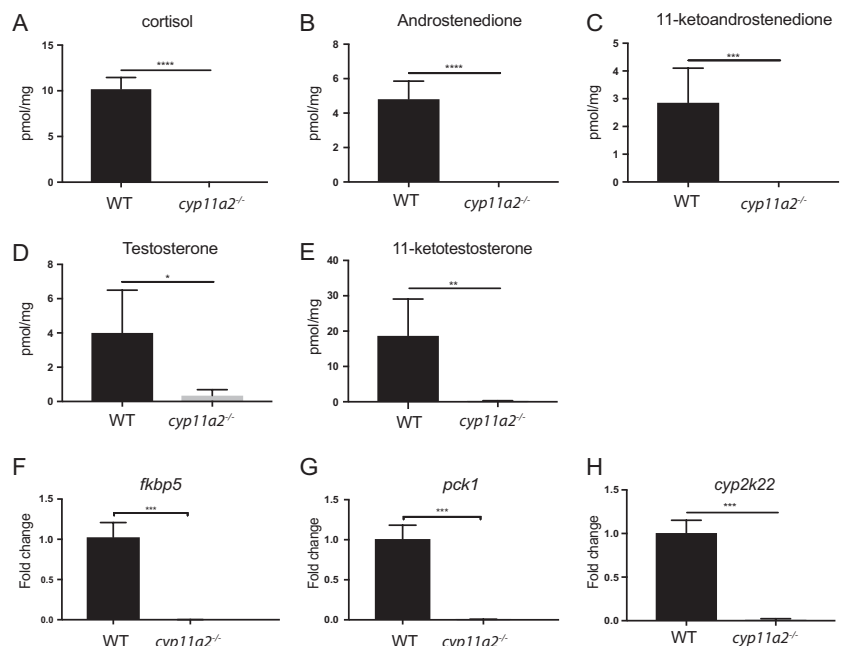

Figure 5

Zebrafish cyp11a2-/- mutant adults have impaired interrenal and gonadal steroid hormone biosynthesis. The concentration of (A) cortisol, sex steroids and their precursors (B) androstenedione, (C)

11-ketoandrostenedione, (D) testosterone, and (E) 11-ketotestosterone were significantly decreased in cyp11a2-/- adult males compared to wild-type siblings $(n=8)$. Transcript levels of (F) $f k b p 5$ and (G) $p c k 1$ were also significantly decreased in cyp $11 a 2^{-/-}$adult liver compared to the livers of wild-type siblings $(n=5)$. $(H)$ Expression of the androgenresponsive gene cyp $2 k 22$ was almost completely extinguished in the liver of cyp11a2-/- adult males $(n=5)$. An unpaired $t$-test was used for all analysis. ${ }^{*} P<0.05 ; * \star P<0.005 ; * \star * P<0.0005 ; * \star * * P<0.0001$.

(Fig. 4B and C). Further analysis on $g h 1$ (growth hormone 1) showed significantly increased expression $(P=0.001)$ in the testis of cyp11a2-/- mutant adults $(n=5)$ but no significant change in $g h 1$ expression in the pituitary $(P=0.1236)$ compared to wild-type siblings $(n=5)$ (Fig. 4D and E).

\section{cyp11a2-/- mutant adults are both glucocorticoid and androgen deficient}

To determine steroid hormone concentrations in adults, the whole bodies of wild-type siblings and cyp11a2-/mutants at $180 \mathrm{dpf}$ (eight biological replicates) were used for LC/MS-MS analysis. Mutation of cyp11a2 resulted in reduced cortisol concentrations $(P<0.0001)$ (Fig. 5A). Systemic glucocorticoid deficiency was confirmed by significantly decreased expression of fkbp5 $(P=0.0006)$ and pck1 $(P=0.0005)$ in the livers of cyp11a2-/- adult animals, compared to their siblings (Fig. 5F and G). Furthermore, loss of cyp11a2 function caused significant reductions in the concentration of androstenedione $(P<0.0001), 11$-ketoandrostenedione $(P=0.0009)$, testosterone $(P=0.0128)$ and the active zebrafish androgen, 11-ketotestosterone $(P=0.0038)$ (Fig. 5B, C, D and E). Transcription of the known 
androgen-responsive gene cyp2k22 was also markedly decreased in the livers of cyp11a2-/- males compared to their wild-type siblings ( $P=0.0003$ ) (Fig. $5 \mathrm{H})$.

\section{Cyp11a2 deficiency causes activation of the hypothalamic-pituitary-gonadal axis (HPG axis)}

In response to the impaired sex steroid biosynthesis, significantly higher levels of $f s h b(P=0.0053)$ and $l h b$ $(P=0.0404)$ transcripts were detected in the pituitary of cyp11a2-/- mutant adults $(n=5)$ compared to wild-type siblings $(n=5)$ (Supplementary Fig. $1 \mathrm{~A}$ and B), which may be caused by lack of negative feedback due to androgen deficiency.

\section{Cyp11a2-/- homozygous adults are infertile}

Fertility of $c y p 11 a 2^{-/-}$fish was first assessed by out-crossing cyp11a2-/- mutants or their wild-type male siblings with an unrelated wild-type female. In four independent experiments, we found that wild-type males $(n=10)$ could successfully fertilize eggs produced by the females at a frequency of between 70-90\%, whereas none of the pairs that included a cyp11a2-/- mutant $(n=10)$ could produce fertilized eggs (Supplementary Fig. 2A). We also examined the breeding behavior of wild-type male siblings $(n=6)$ and cyp11a2 $2^{--}$mutants $(n=6)$ out-crossed with unrelated wild-type females. Wild-type males exhibited typical breeding behaviors, that is chasing wild-type females, making intimate contacts and inducing spawning (Supplementary Fig. 2B and C). However, cyp11a2-/mutants exhibited greatly reduced levels of chasing and intimate contact with wild-type females (Supplementary Fig. 2B and C). Consequently, this resulted in failure of spawning induction in females.

Sperm concentration was then examined and found significantly decreased in cyp11a2-/- mutants compared to wild-type siblings $(P=0.0071)$ (Fig. 6A). Fertility of cyp11a2-/- mutants was further assessed by IVF. Interestingly, sperm from the dissected testis $(n=5)$ could not fertilize any wild-type eggs $(P<0.0001)$, compared to $50-90 \%$ fertilization rate by wild-type sperm (Fig. 6B).

Taken together, our results suggested that loss of cyp11a2 function considerably impairs the fertility of adult males.

\section{Homozygous cyp11a2-/- mutant fish exhibit defective spermatogenesis and disorganized testes}

The finding of a low sperm count and the absence of breeding behaviors in $c y p 11 \mathrm{a}^{-/-}$mutants led us to examine testicular structures and functions. Histological analysis using H\&E staining showed well-organized testes in wildtype siblings. These testes had well-defined seminiferous tubules separated by thin strands of interstitial connective tissue. Spermatozoa were located in the center of each tubular lumen, with spermatids, spermatocytes and
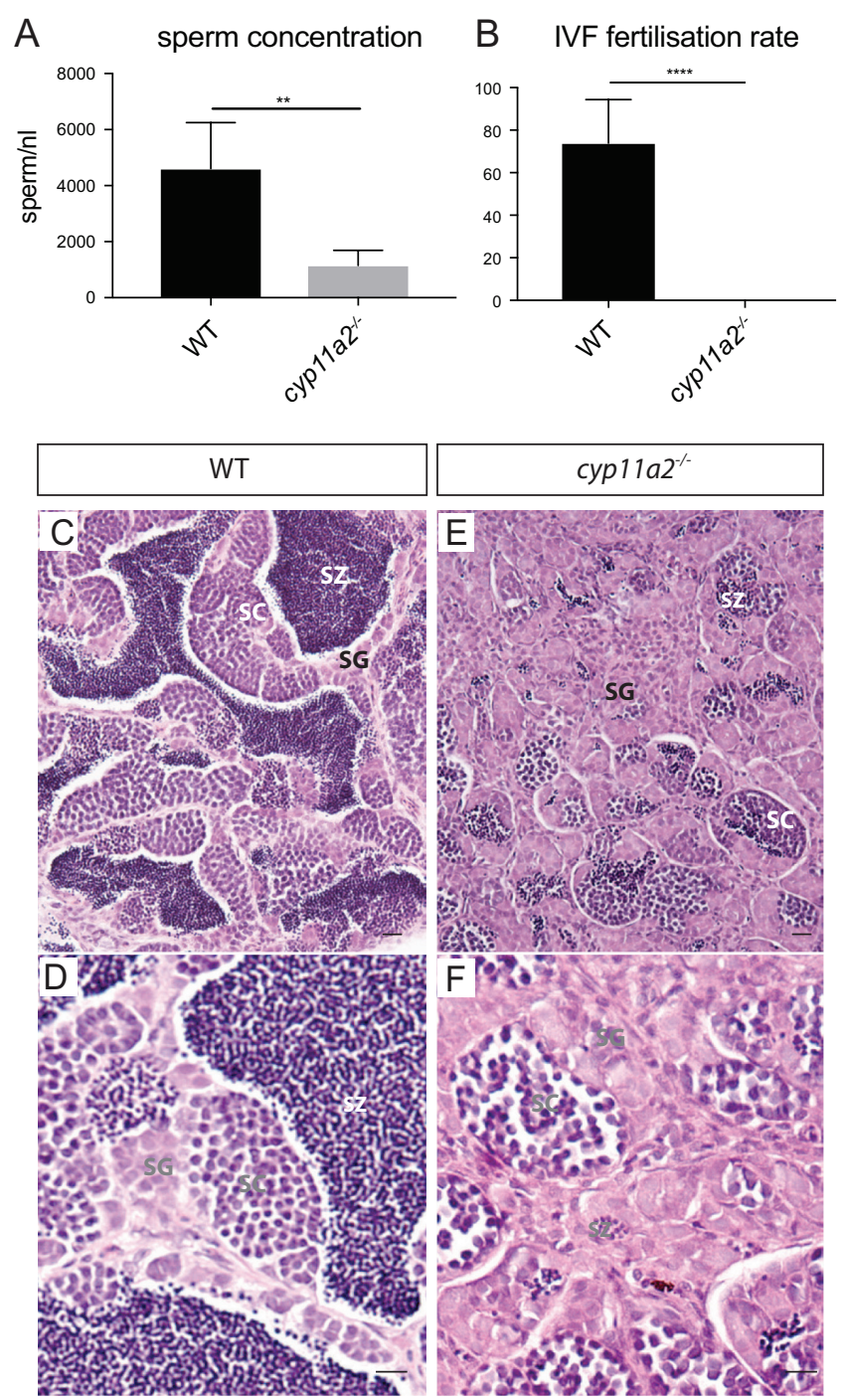

\section{Figure 6}

Cyp11a2-/- homozygous male adults are infertile. (A) Sperm concentration was markedly reduced in cyp11a2-/- mutant males $(n=4)$. (B) Sperm from the dissected testis of the cyp11a2-/- mutants $(n=5)$ could not fertilize any wild-type eggs, compared to $50-90 \%$ fertilization rate by wild-type sperm. (C, D, E and F) Histological analysis of testes at $180 \mathrm{dpf}$ showed that cyp11a2-/- mutants (E and F) had disorganized seminiferous tubules with a barely distinguishable central lumen compared to the testes of wild-type males ( $C$ and $D)$. Spermatogonia were the main cell type found in the mutant testes, with some spermatocytes and a very few spermatozoa also present. SG, spermatogonia; SC, spermatocyte; SZ, spermatozoa. Scale bar: $10 \mu \mathrm{m}$. An unpaired $t$-test was used for the analysis. ${ }^{*} P<0.005, * * * * P<0.0001$. 
spermatogonia located more peripherally, toward the basement membrane (Fig. 6C and D).

By contrast, the testes of cyp11a2-/- mutants were markedly disorganized and adjacent seminiferous tubule cross-sections were difficult to distinguish from one another. For each tubule cross-section, the central lumen was difficult to identify, and mature spermatozoa were very rarely found within the testis. Spermatogonia were the main cell type found in the testes of cyp11a2-/- mutants, with spermatocytes being the second dominant cell type (Fig. 6E and F). Interestingly, there was no difference in gonadosomatic index between cyp11a2-/- mutant and wild-type siblings at $180 \mathrm{dpf}$ (Supplementary Fig. 2D).

\section{Cyp11a2 deficiency causes downregulation of key genes involved in testis development and spermatogenesis}

To investigate the molecular mechanisms underlying the phenotypic defects observed, we used qRT-PCR to examine the transcription of genes involved in testis development and spermatogenesis.

Doublesex and mab-3-related transcription factor 1 (dmrt1) is a key regulator of testis development, and anti-Mullerian hormone $(\mathrm{amh})$ is a pro-testis hormone during zebrafish development. Interestingly, the expression of both genes was decreased in the testes of cyp11a2-/- mutants (dmrt1, $P=0.0151$; amh, $P=0.0002$ ) (Fig. 7A and B). Furthermore, SRY-box 9a (sox9a), a pro-male transcription factor involved in ovary-testis transition in zebrafish, is also significantly downregulated $(P=0.0249)$ (Fig. 7C).
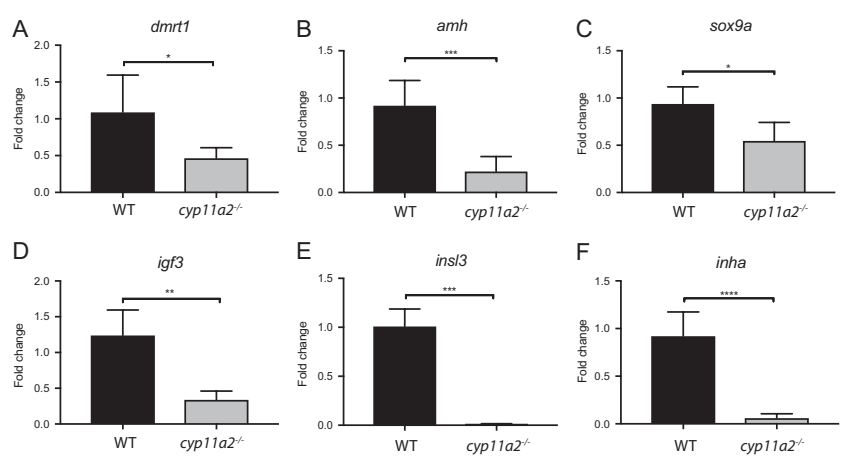

Figure 7

Expression of pro-testis and spermatogenic genes was downregulated in the testes of cyp11a2-/- zebrafish. (A, B, C, D, E and F) Relative expression of $d m r t 1, a m h$, sox $9 a$, igf3, ins/3 and inha were significantly decreased in the testes of homozygous mutants compared to wild-type siblings $(n=5)$. An unpaired $t$-test was used for all analysis. ${ }^{*} P<0.05$; ${ }^{*} P<0.005$; $\star * \star P<0.0005, * * * * P<0.0001$.
Insulin-like growth factor 3 (igf3) and insulin-like peptide 3 (insl3) are both involved in the proliferation and differentiation of type A spermatogonia in the early stages of spermatogenesis (Crespo et al. 2016, Safian et al. 2016). Significant downregulation of igf3 $(P=0.001)$ (Fig. 7D) and insl3 $(P=0.0006)$ were found in cyp11a2-/- testes (Fig. 7E), consistent with the findings of decreased sperm quantity. The transcript level of inhibin alpha (inha) was almost completely extinguished in testes from cyp11a2-/mutants $(P<0.0001)$ (Fig. 7F).

To further investigate molecular mechanisms of the decreased expression of igf 3 and insl3, we examined the expression of $f s h r$ and $l h c g r$ in the testes of both mutant and wild-type sibling adults. However, no significant difference was detected for either $f s h r(P=0.2481)$ or $l h c g r$ $(P=0.1372)$ (Supplementary Fig. 1C and D).

\section{Differential expression of spermatogenesis markers in cyp11a2 homozygous mutants}

To investigate how loss of Cyp11a2 function impacts spermatogenesis, we measured the expression of germ cell markers at several stages of spermatogenesis. nanos2 is expressed in type A undifferentiated spermatogonia, while piwil1 is expressed in all type A spermatogonia (Beer \& Draper 2013, Safian et al. 2016). We found a significant upregulation of nanos $2(P=0.0004)$ and piwil1 $(P=0.022)$ expression in the testes of $c y p 11 a 2^{-/-}$fish. No change was found in the expression of dazl, a marker of type B spermatogonia $(P=0.8219)$ (Safian et al. 2016). Interestingly, expression of spermatocyte marker sycp3 $(P=0.0012)$ and spermatid marker odf3b $(P=0.0003)$ was significantly downregulated (Fig. 8A, B, C, D and E) (Ozaki et al. 2011, Tang et al. 2018). The expression profile is consistent with the assessment of sperm quality and quantity, suggesting decreased numbers of late stage germ cells and accumulation of germ cell precursors.

\section{Discussion}

Previous data by our group and others (Hsu et al. 2009, Parajes et al. 2013) suggested that cyp11a1 is expressed during early development stages from zygote to $24 \mathrm{hpf}$, while the expression of cyp11a2 becomes continuously prominent just before de novo (48 hpf) and HPI-mediated (96 hpf) cortisol synthesis commences (Parajes et al. 2013, Weger et al. 2018). Further in situ hybridization analysis showed the expression of cyp11a2 is restricted to the interrenal at $120 \mathrm{hpf}$, whereas no expression of cyp11a1 

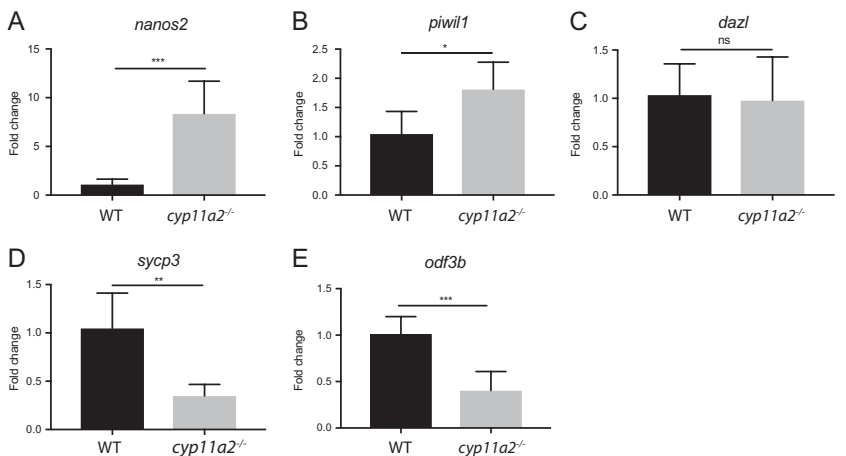

Figure 8

Spermatogenesis markers were differentially expressed in the testes of cyp 11a2-/- mutant and wild-type adults. Expression of (A) nanos2 and (B) piwil1, which are markers for type $A_{\text {und }}$ spermatogonia and all type $A$ spermatogonia, respectively, was significantly increased in the testes of homozygous mutants $(n=5)$. (C) Expression of dazl, a marker of type B spermatogonia, was not changed $(n=5)$. Expression of the spermatocyte marker (D) sycp3, and the spermatid marker, (E) odf3b was significantly downregulated $(n=5)$. An unpaired $t$-test was used for all analysis. $\star P<0.05 ; * * P<0.005 ; * \star \star P<0.0005$.

was found (Weger et al. 2018). Functional analysis using transient morpholino knockdown in larvae revealed distinct roles between the two genes. cyp11a1 is required for early embryonic development, which is consistent with observations in other reports (Goldstone et al. 2010). cyp11a2 is essential for de novo steroidogenesis in zebrafish larvae and appeared to be the functional equivalent of human CYP11A1 (Parajes et al. 2013). In this study, we confirmed previously reported results and clarified the role of Cyp11a2 in adult zebrafish using stable null-allele lines.

\section{Disruption of cyp11a2 leads to glucocorticoid deficiency}

Previous morpholino knockdown of cyp11a2 showed various levels of phenotypic abnormalities including craniofacial defects, pericardiac oedema and absence of swim bladder (Parajes et al. 2013). Similar phenotypes were found in loss of $f f 1 b$ (ortholog of mammalian steroidogenic factor 1) or dax1, but all the studies were based on morpholinos (Chai et al. 2003, Zhao et al. 2006). In contrast, these phenotypes were absent in our newly generated cyp11a2-/- knockout lines and such phenotypes have also not been observed in our published glucocorticoid-deficient lines due to 21-hydroxylase (Eachus et al. 2017) or ferredoxin deficiency (Griffin et al. 2016). Morpholinos are well known for their off-target effects (Eisen \& Smith 2008) and thus the previously described changes attributed to glucocorticoid deficiency
(Parajes et al. 2013) are more likely caused by morpholino toxicity (Stainier et al. 2017).

Our cyp11a2-/- mutants displayed typical characteristics of glucocorticoid deficiency. VBA is a glucocorticoiddependent neuroendocrine response mediated via the glucocorticoid receptor (Muto et al. 2005, 2013). Impaired VBA has been previously described in several zebrafish lines with defective glucocorticoid signaling (Griffin et al. 2016, Eachus et al. 2017, Facchinello et al. 2017). The VBA analysis of larvae at $120 \mathrm{hpf}$ revealed that about $93 \%$ of the

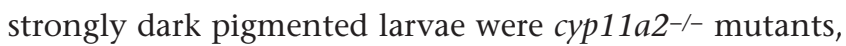
suggesting impaired glucocorticoid biosynthesis.

Adrenal insufficiency causes an upregulation of the hypothalamus-pituitary-adrenal axis in humans due to a disruption of the negative HPA feedback (Liyanarachchi et al. 2017). Our cyp11a2-/- mutants exhibited an upregulation of the HPI axis as indicated by increased pomca expression (Fig. 3). This is consistent with other zebrafish models of glucocorticoid resistance (Ziv et al. 2013, Lin et al. 2015) and glucocorticoid deficiency (Griffin et al. 2016, Eachus et al. 2017) due to loss of negative feedback to the pituitary and hypothalamus.

The significantly decreased concentration of cortisol in the VBA- larvae clearly demonstrates the requirement for Cyp11a2 to enable glucocorticoid biosynthesis, which is consistent with our previous morpholino knockdown study (Parajes et al. 2013). However, some baseline cortisol production was detected in VBA- larvae. The most plausible explanation is that a small proportion of wild-type or heterozygous siblings are also VBA- but designated as cyp11a2-- mutant larvae. This limitation to the use of VBA as a genotyping proxy has been highlighted previously (Eachus et al. 2017). However, the lack of detectable concentrations of cortisol in cyp11a2-/adults confirmed that they are glucocorticoid deficient. To further validate this model of glucocorticoid deficiency, we subjected the larvae to osmotic stress, which has previously been used to induce cortisol production (Weger et al. 2012). However, there was no transcriptional increase of either $f k b p 5$ or $p c k 1$ in the $c y p 11 a 2^{-/-}$mutant larvae under osmotic stress, further demonstrating a block in glucocorticoid biosynthesis.

\section{Disruption of cyp11a2 leads to sex steroid deficiency and all-male fish with feminized secondary sex characteristics (SSCs)}

Sex steroids are required for sexual development and are also responsible for the maintenance of established sex phenotypes (Goppert et al. 2016). We found 
significantly reduced concentrations of the sex steroid precursor androstenedione, the active zebrafish androgen 11-ketotestosterone, and testosterone in cyp11a2-/- adult mutants. All cyp11a2-/- mutant zebrafish developed testes. Previous studies on cyp19a1a and cyp17a1 in zebrafish have shown all-male phenotypes (Lau et al. 2016, Zhai et al. 2018). Therefore, our results suggest that the impaired biosynthesis of sex steroids in cyp $11 a 2^{-/-}$mutant zebrafish is responsible for their all-male phenotype.

Cyp11a2-deficient male zebrafish mainly exhibited feminized SSCs, including female body pigmentation and a rounded body shape. In teleost fish, androgens, especially 11-ketotestosterone, are important for testicular development and for the development of SSCs (Borg 1994). Both testosterone and 11-ketotestosterone have high affinity and transactivation potency for the androgen receptor (Ar) in zebrafish (de Waal et al. 2008, Hossain et al. 2008). Similarly, Ar-deficient zebrafish males also exhibited female-type coloration and body shape (Crowder et al. 2018, Yu et al. 2018). Moreover, feminized SSCs were also demonstrated in the Cyp17a1-deficient zebrafish males; however, this androgen-deficient line exhibited a slender body shape as found in wild-type males (Zhai et al. 2018). Taken together, these results suggest that the development of male SSCs requires appropriate androgen exposure. However, the cause of observed phenotypic variability in different androgendeficient lines remains to be elucidated.

\section{cyp11a2-/- mutants exhibit male infertility}

Our cyp11a2-/- mutants failed to exhibit male-typical chasing activity and the ability to induce female spawning during natural breeding. Similarly, Ar-deficient zebrafish and androgen-deficient cyp17a1-knockout zebrafish exhibit defective breeding behaviors (Crowder et al. 2018, Tang et al. 2018, Yu et al. 2018, Zhai et al. 2018). $\mathrm{Ar}$ is necessary for zebrafish fertility, and male mating behaviors are likely to be regulated by androgen signaling. Further fertility assessment by sperm counting revealed significantly reduced sperm concentrations in cyp11a2-/mutants, indicating impaired spermatogenesis. A lower amount of mature spermatozoa was also observed in the testes of zebrafish ar-/- mutants (Crowder et al. 2018, Tang et al. 2018, Yu et al. 2018). Interestingly, normal spermatogenesis was found in Cyp17a1-deficient zebrafish mutants, possibly due to compensation by Cyp17a2 (Zhai et al. 2018). However, biochemical analysis suggested zebrafish Cyp17a2 cannot catalyze the 17,20lyase reactions that yield to DHEA and androstenedione (Pallan et al. 2015). Progesterone has been suggested to function in spermatogonial cell proliferation and spermatogenesis in teleosts (Liu et al. 2014, Wang et al. 2016). Moreover, the specificity of the interactions between Ar and its ligands are not fully characterized in teleosts. The development of relatively normal testes with mature spermatozoa in cyp17a1 mutant animals might be explained by some affinity of Ar for progesterone (de Waal et al. 2008).

In mammals, androgen signaling is believed to be required for testis development and spermatogenesis. A murine Cyp11a1 deletion model had testes with disorganized seminiferous tubules containing only spermatocytes arrested at the meiotic stage (Hu et al. 2002). Our histological analysis of the testes from cyp11a2-/mutants showed poorly defined seminiferous tubules without a distinguishable central lumen. Moreover, the seminiferous tubules mainly contained spermatogonia and very few spermatocytes or mature spermatozoa. Similarly, zebrafish ar $^{-1-}$ mutants also exhibit disorganized seminiferous tubules replete with spermatogonia and spermatocytes but deficient in mature spermatozoa (Crowder et al. 2018, Yu et al. 2018).

Whilst the molecular mechanism underlying the severe disruption of spermatogenesis in Cyp11a2-deficient zebrafish remains elusive, our observations of markedly reduced igf 3 and insl 3 expression may contribute to the accumulation of early spermatogonial cells as Igf 3 and Insl3 are both involved in the proliferation and differentiation of type A spermatogonia (Assis et al. 2016, Crespo et al. 2016, Safian et al. 2016). Downregulation of igf3 and insl3 was previously reported in ar-null mutants (Tang et al. 2018). Moreover, unchanged level of dazl expression, a marker of type B spermatogonia, suggests normal differentiation from type A to type B spermatogonia. On the other hand, the reduced expression of the spermatocyte marker sycp3 and spermatid marker $o d f 3 b$ indicate that the transition into and/or completion of meiosis are compromised.

In this study, we generated cyp11a2-knockout animals by employing CRISPR/Cas9 gene editing technology. These cyp11a2-null mutant zebrafish are deficient in both glucocorticoid and sex steroids. We demonstrated the phenotypic consequences of the combined steroid hormone deficiencies. This work thus establishes a requirement for Cyp11a2 in adrenal and gonadal steroidogenesis in zebrafish. 


\section{Supplementary materials}

This is linked to the online version of the paper at https://doi.org/10.1530/ JOE-19-0384.

\section{Declaration of interest}

The authors declare that there is no conflict of interest that could be perceived as prejudicing the impartiality of the research reported.

\section{Funding}

This work was supported by the International Fund Congenital Adrenal Hyperplasia 2017 research grant (awarded to N P K and V T C), the Deutsche Forschungsgemeinschaft (KR 3363/3-1 to N P K) and the Society for Endocrinology (early career grant to $\mathrm{N} \mathrm{L}$ ).

\section{Author contribution statement}

V T Cunliffe and N P Krone contributed equally to this work.

\section{Acknowledgements}

The authors would like to thank all the aquarium staff in the University of Sheffield for maintenance of fish stocks, and Maggie Glover for help with histological analysis.

\section{References}

Assis LH, Crespo D, Morais RD, Franca LR, Bogerd J \& Schulz RW 2016 INSL3 stimulates spermatogonial differentiation in testis of adult zebrafish (Danio rerio). Cell and Tissue Research 363 579-588. (https:// doi.org/10.1007/s00441-015-2213-9)

Beer RL \& Draper BW 2013 nanos3 maintains germline stem cells and expression of the conserved germline stem cell gene nanos 2 in the zebrafish ovary. Developmental Biology 374 308-318. (https://doi. org/10.1016/j.ydbio.2012.12.003)

Borg B 1994 Androgens in teleost fishes. Comparative Biochemistry and Physiology 109 219-245. (https://doi.org/10.1016/07428413(94)00063-G)

Chai C, Liu YW \& Chan WK 2003 Ff1b is required for the development of steroidogenic component of the zebrafish interrenal organ. Developmental Biology 260 226-244. (https://doi.org/10.1016/s00121606(03)00219-7)

Crespo D, Assis LHC, Furmanek T, Bogerd J \& Schulz RW 2016 Expression profiling identifies Sertoli and Leydig cell genes as Fsh targets in adult zebrafish testis. Molecular and Cellular Endocrinology 437 237-251. (https://doi.org/10.1016/j.mce.2016.08.033)

Crowder CM, Lassiter CS \& Gorelick DA 2018 Nuclear androgen receptor regulates testes organization and oocyte maturation in zebrafish. Endocrinology 159 980-993. (https://doi.org/10.1210/ en.2017-00617)

de Waal PP, Wang DS, Nijenhuis WA, Schulz RW \& Bogerd J 2008 Functional characterization and expression analysis of the androgen receptor in zebrafish (Danio rerio) testis. Reproduction 136 225-234. (https://doi.org/10.1530/REP-08-0055)

Dickmeis T 2009 Glucocorticoids and the circadian clock. Journal of Endocrinology 200 3-22. (https://doi.org/10.1677/JOE-08-0415)
Dranow DB, Tucker RP \& Draper BW 2013 Germ cells are required to maintain a stable sexual phenotype in adult zebrafish. Developmental Biology 376 43-50. (https://doi.org/10.1016/j.ydbio.2013.01.016)

Eachus H, Zaucker A, Oakes JA, Griffin A, Weger M, Guran T, Taylor A, Harris A, Greenfield A, Quanson JL, et al. 2017 Genetic disruption of 21-hydroxylase in zebrafish causes interrenal hyperplasia. Endocrinology 158 4165-4173. (https://doi.org/10.1210/en.2017-00549)

Eisen JS \& Smith JC 2008 Controlling morpholino experiments: don't stop making antisense. Development 135 1735-1743. (https://doi. org/10.1242/dev.001115)

Facchinello N, Skobo T, Meneghetti G, Colletti E, Dinarello A, Tiso N, Costa R, Gioacchini G, Carnevali O, Argenton F, et al. 2017 nr3c1 null mutant zebrafish are viable and reveal DNA-binding-independent activities of the glucocorticoid receptor. Scientific Reports $\mathbf{7} 4371$. (https://doi.org/10.1038/s41598-017-04535-6)

Goldstone JV, McArthur AG, Kubota A, Zanette J, Parente T, Jonsson ME, Nelson DR \& Stegeman JJ 2010 Identification and developmental expression of the full complement of cytochrome P450 genes in zebrafish. BMC Genomics 11 643. (https://doi.org/10.1186/1471-216411-643)

Goppert C, Harris RM, Theis A, Boila A, Hohl S, Ruegg A, Hofmann HA, Salzburger W \& Bohne A 2016 Inhibition of aromatase induces partial sex change in a cichlid fish: distinct functions for sex steroids in brains and gonads. Sexual Development 10 97-110. (https://doi. org/10.1159/000445463)

Griffin A, Parajes S, Weger M, Zaucker A, Taylor AE, O’Neil DM, Muller F \& Krone N 2016 Ferredoxin 1b (Fdx1b) is the essential mitochondrial redox partner for cortisol biosynthesis in zebrafish. Endocrinology 157 1122-1134. (https://doi.org/10.1210/en.2015-1480)

Hossain MS, Larsson A, Scherbak N, Olsson PE \& Orban L 2008 Zebrafish androgen receptor: isolation, molecular, and biochemical characterization. Biology of Reproduction 78 361-369. (https://doi. org/10.1095/biolreprod.107.062018)

Hruscha A, Krawitz P, Rechenberg A, Heinrich V, Hecht J, Haass C \& Schmid B 2013 Efficient CRISPR/Cas9 genome editing with low offtarget effects in zebrafish. Development 140 4982-4987. (https://doi. org/10.1242/dev.099085)

Hsu HJ, Lin JC \& Chung BC 2009 Zebrafish cyp11a1 and hsd3b genes: structure, expression and steroidogenic development during embryogenesis. Molecular and Cellular Endocrinology 312 31-34. (https://doi.org/10.1016/j.mce.2009.07.030)

$\mathrm{Hu}$ MC, Hsu NC, El Hadj NB, Pai CI, Chu HP, Wang CK \& Chung BC 2002 Steroid deficiency syndromes in mice with targeted disruption of Cyp11a1. Molecular Endocrinology 16 1943-1950. (https://doi. org/10.1210/me.2002-0055)

Jing R, Huang C, Bai C, Tanguay R \& Dong Q 2009 Optimization of activation, collection, dilution, and storage methods for zebrafish sperm. Aquaculture 290 165-171. (https://doi.org/10.1016/j. aquaculture.2009.02.027)

Kroeger Jr PT, Poureetezadi SJ, McKee R, Jou J, Miceli R \& Wingert RA 2014 Production of haploid zebrafish embryos by in vitro fertilization. Journal of Visualized Experiments 89 51708. (https://doi. org/10.3791/51708)

Lau ES, Zhang Z, Qin M \& Ge W 2016 Knockout of zebrafish ovarian aromatase gene (cyp19a1a) by TALEN and CRISPR/Cas9 leads to allmale offspring due to failed ovarian differentiation. Scientific Reports 6 37357. (https://doi.org/10.1038/srep37357)

Lin JC, Hu S, Ho PH, Hsu HJ, Postlethwait JH \& Chung BC 2015 Two zebrafish hsd3b genes are distinct in function, expression, and evolution. Endocrinology 156 2854-2862. (https://doi.org/10.1210/en.2014-1584)

Liu YW 2007 Interrenal organogenesis in the zebrafish model. Organogenesis 3 44-48. (https://doi.org/10.4161/org.3.1.3965)

Liu G, Luo F, Song Q, Wu L, Qiu Y, Shi H, Wang D \& Zhou L 2014 Blocking of progestin action disrupts spermatogenesis in Nile tilapia (Oreochromis niloticus). Journal of Molecular Endocrinology 53 57-70. (https://doi.org/10.1530/JME-13-0300) 
Liyanarachchi K, Ross R \& Debono M 2017 Human studies on hypothalamo-pituitary-adrenal (HPA) axis. Best Practice and Research: Clinical Endocrinology and Metabolism 31 459-473. (https://doi. org/10.1016/j.beem.2017.10.011)

Lohr H \& Hammerschmidt M 2011 Zebrafish in endocrine systems: recent advances and implications for human disease. Annual Review of Physiology 73 183-211. (https://doi.org/10.1146/annurevphysiol-012110-142320)

Miller WL \& Auchus RJ 2011 The molecular biology, biochemistry, and physiology of human steroidogenesis and its disorders. Endocrine Reviews 32 81-151. (https://doi.org/10.1210/er.2010-0013)

Muthu V, Eachus H, Ellis P, Brown S \& Placzek M 2016 Rx3 and Shh direct anisotropic growth and specification in the zebrafish tuberal/anterior hypothalamus. Development 143 2651-2663. (https://doi.org/10.1242/ dev.138305)

Muto A, Orger MB, Wehman AM, Smear MC, Kay JN, Page-McCaw PS, Gahtan E, Xiao T, Nevin LM, Gosse NJ, et al. 2005 Forward genetic analysis of visual behavior in zebrafish. PLoS Genetics 1 e66. (https:// doi.org/10.1371/journal.pgen.0010066)

Muto A, Taylor MR, Suzawa M, Korenbrot JI \& Baier H 2013 Glucocorticoid receptor activity regulates light adaptation in the zebrafish retina. Frontiers in Neural Circuits 7 145. (https://doi. $\operatorname{org} / 10.3389 /$ fncir.2013.00145)

O’Reilly MW, Kempegowda P, Jenkinson C, Taylor AE, Quanson JL, Storbeck KH \& Arlt W 2017 11-Oxygenated C19 steroids are the predominant androgens in polycystic ovary syndrome. Journal of Clinical Endocrinology and Metabolism 102 840-848. (https://doi. org/10.1210/jc.2016-3285)

Ozaki Y, Saito K, Shinya M, Kawasaki T \& Sakai N 2011 Evaluation of Sycp3, Plzf and cyclin B3 expression and suitability as spermatogonia and spermatocyte markers in zebrafish. Gene Expression Patterns 11 309-315. (https://doi.org/10.1016/j.gep.2011.03.002)

Pallan PS, Nagy LD, Lei L, Gonzalez E, Kramlinger VM, Azumaya CM, Wawrzak Z, Waterman MR, Guengerich FP \& Egli M 2015 Structural and kinetic basis of steroid 17alpha,20-lyase activity in teleost fish cytochrome P450 17A1 and its absence in cytochrome P450 17A2. Journal of Biological Chemistry 290 3248-3268. (https://doi. org/10.1074/jbc.M114.627265)

Parajes S, Griffin A, Taylor AE, Rose IT, Miguel-Escalada I, Hadzhiev Y, Arlt W, Shackleton C, Muller F \& Krone N 2013 Redefining the initiation and maintenance of zebrafish interrenal steroidogenesis by characterizing the key enzyme cyp11a2. Endocrinology $1542702-$ 2711. (https://doi.org/10.1210/en.2013-1145)

Safian D, Morais RD, Bogerd J \& Schulz RW 2016 Igf binding proteins protect undifferentiated spermatogonia in the zebrafish testis against excessive differentiation. Endocrinology 157 4423-4433. (https://doi. org/10.1210/en.2016-1315)

Schilling TF 2002 The morphology of larval and adult zebrafish. In Zebrafish, 1st ed. Eds C Nüsslein-Volhard \& R Dahm. Oxford, UK: Oxford University Press. (https://doi.org/10.1017/ S0016672303216384)

Stainier DYR, Raz E, Lawson ND, Ekker SC, Burdine RD, Eisen JS, Ingham PW, Schulte-Merker S, Yelon D, Weinstein BM, et al. 2017 Guidelines for morpholino use in zebrafish. PLoS Genetics $\mathbf{1 3}$ e1007000. (https://doi.org/10.1371/journal.pgen.1007000)

Talbot JC \& Amacher SL 2014 A streamlined CRISPR pipeline to reliably generate zebrafish frameshifting alleles. Zebrafish 11 583-585. (https://doi.org/10.1089/zeb.2014.1047)
Tang H, Chen Y, Wang L, Yin Y, Li G, Guo Y, Liu Y, Lin H, Cheng CHK \& Liu X 2018 Fertility impairment with defective spermatogenesis and steroidogenesis in male zebrafish lacking androgen receptor. Biology of Reproduction 98 227-238. (https://doi.org/10.1093/biolre/ iox 165)

Thisse C \& Thisse B 2008 High-resolution in situ hybridization to wholemount zebrafish embryos. Nature Protocols 3 59-69. (https://doi. org/10.1038/nprot.2007.514)

To TT, Hahner S, Nica G, Rohr KB, Hammerschmidt M, Winkler C \& Allolio B 2007 Pituitary-interrenal interaction in zebrafish interrenal organ development. Molecular Endocrinology 21 472-485. (https://doi. org/10.1210/me.2006-0216)

Tokarz J, Moller G, de Angelis MH \& Adamski J 2013 Zebrafish and steroids: what do we know and what do we need to know? Journal of Steroid Biochemistry and Molecular Biology 137 165-173. (https://doi. $\operatorname{org} / 10.1016 /$ j.jsbmb.2013.01.003)

Tokarz J, Moller G, Hrabe de Angelis M \& Adamski J 2015 Steroids in teleost fishes: a functional point of view. Steroids 103 123-144. (https://doi.org/10.1016/j.steroids.2015.06.011)

Wang C, Liu D, Chen W, Ge W, Hong W, Zhu Y \& Chen SX 2016 Progestin increases the expression of gonadotropins in pituitaries of male zebrafish. Journal of Endocrinology 230 143-156. (https://doi. org/10.1530/JOE-16-0073)

Weger BD, Weger M, Nusser M, Brenner-Weiss G \& Dickmeis T 2012 A chemical screening system for glucocorticoid stress hormone signaling in an intact vertebrate. ACS Chemical Biology 7 1178-1183. (https://doi.org/10.1021/cb3000474)

Weger M, Diotel N, Weger BD, Beil T, Zaucker A, Eachus HL, Oakes JA, do Rego JL, Storbeck KH, Gut P, et al. 2018 Expression and activity profiling of the steroidogenic enzymes of glucocorticoid biosynthesis and the fdx1 co-factors in zebrafish. Journal of Neuroendocrinology $\mathbf{3 0}$ e12586. (https://doi.org/10.1111/jne.12586)

Westerfield M 2000 The Zebrafish Book: A Guide for the Laboratory Use of Zebrafish (Danio rerio), 4th ed. Eugene, OR, USA: University of Oregon Press.

World Health Organization 2010 WHO Laboratory Manual for the Examination and Processing of Human Semen, 5th ed. Geneva, Switzerland: WHO.

Yossa R, Sarker PK, Proulx E, Saxena V, Ekker M \& Vandenberg GW 2013 A practical approach for sexing zebrafish (Danio rerio). Journal of Applied Aquaculture 25 148-153. (https://doi.org/10.1080/10454438. 2013.792170)

Yu G, Zhang D, Liu W, Wang J, Liu X, Zhou C, Gui J \& Xiao W 2018 Zebrafish androgen receptor is required for spermatogenesis and maintenance of ovarian function. Oncotarget 9 24320-24334. (https:// doi.org/10.18632/oncotarget.24407)

Zhai G, Shu T, Xia Y, Lu Y, Shang G, Jin X, He J, Nie P \& Yin Z 2018 Characterization of sexual trait development in cyp17a1-deficient zebrafish. Endocrinology 159 3549-3562. (https://doi.org/10.1210/ en.2018-00551)

Zhao Y, Yang Z, Phelan JK, Wheeler DA, Lin S \& McCabe ER 2006 Zebrafish dax 1 is required for development of the interrenal organ, the adrenal cortex equivalent. Molecular Endocrinology 20 2630-2640. (https://doi.org/10.1210/me.2005-0445)

Ziv L, Muto A, Schoonheim PJ, Meijsing SH, Strasser D, Ingraham HA, Schaaf MJ, Yamamoto KR \& Baier H 2013 An affective disorder in zebrafish with mutation of the glucocorticoid receptor. Molecular Psychiatry 18 681-691. (https://doi.org/10.1038/mp.2012.64)

Received in final form 25 October 2019

Accepted 5 November 2019

Accepted Manuscript published online 6 November 2019 https://joe.bioscientifica.com https://doi.org/10.1530/JOE-19-0384
(C) 2020 Society for Endocrinology Published by Bioscientifica Ltd. Printed in Great Britain 